\title{
Micro-force measurement with pre-curvature long-period fiber grating-based sensor
}

\author{
Walter S. J. Ferreira ${ }^{1,2}$, Paulo S. S. dos Santos ${ }^{3,5}$, Paulo Caldas ${ }^{3,4 *}$, Pedro A. S. Jorge ${ }^{3,5}$, João M. S. Sakamoto ${ }^{2,1}$ \\ ${ }^{1}$ Technological Institute of Aeronautics [Instituto Tecnológico de Aeronáutica - ITA], Praça Mal. Eduardo Gomes, 50, São José dos Campos-SP, 12228-900, Brazil. \\ ${ }^{2}$ Institute for Advanced Studies [Instituto de Estudos Avançados - IEAv], Trevo Cel. Av. José A. A. do Amarante, 01, São José dos Campos- SP, 12228-001, Brazil. \\ ${ }^{3}$ INESC TEC - Institute for Systems and Computer Engineering, Technology and Science, 4200-465 Porto, Portugal. \\ ${ }^{4}$ Escola Superior de Tecnologia e Gestão, Instituto Politécnico de Viana do Castelo Rua Escola Industrial e Comercial de Nun'Álvares, n. 34 4900-347 Viana do Castelo \\ ${ }^{5}$ Departamento de Física e Astronomia da Faculdade de Ciências da Universidade do Porto, Rua do Campo Alegre 687, 4169-007 Porto, Portugal
}

\begin{abstract}
In this work, a long-period fiber grating (LPG) based sensor was evaluated as a sensing device for micro-force measurement, in the order of micro Newtons. It was used an LPG fabricated by arc-inducted technique in a SMF-28 standard optical fiber. The optical fiber was fixed between two clamps with a separation of $150 \mathrm{~mm}$ with the middle of the LPG located at the center. Characterizations were performed in terms of temperature, curvature and strain. The grating was then used as a micro-force sensor by means of both curvature and strain, induced by a hung mass in a stretched fiber. Furthermore, the evaluation of a precurvature LPG was performed to assess if an increase of sensitivity is achieved. Micro-force sensitivity achieved with the stretched LPG was $1.41 \mathrm{~nm} / \mathrm{mN}$ and it was demonstrated that its sensitivity can be enhanced to $5.14 \mathrm{~nm} / \mathrm{mN}$ with a pre-curvature of $2.2 \mathrm{~m}^{-1}$ applied to the $\mathrm{LPG}$, achieving a spectral resolution of at least $15.6 \mu \mathrm{N}$.
\end{abstract}

\section{Introduction}

The research field in optical fiber sensors (OFS) has been steadily growing, mainly because OFS presents interesting characteristics, such as resistance to most common chemicals, lightweight, inherently small form factors, and capability to withstand high temperatures, as well as their immunity to electromagnetic interference. Some of these OFS already have established a firm position as industry solutions, used in hard-toreach areas, performing, for example, corrosion sensing, or in aeronautics and even space industry where fiber Bragg grating (FBG) has been already passed the proof-of-principle stage and is now an established technology [1]-[4].

Apart from FBG, another kind of grating, with well-known characteristics is long-period fiber grating (LPG). This kind of grating is easy to fabricate in cost-effective ways, using, for example, electric-arc discharges. These gratings present some advantages over FBGs, such as refractive index (RI) sensing or low back-reflection. The light is coupled from the fundamental forwarding propagating $\mathrm{LP}_{01}$ core mode to higher-order forward propagating cladding modes [5]-[7] by applying periodic modulations on the fiber core, using phase masks or point-by-point markings (with femtosecond lasers, $\mathrm{CO}_{2}$ lasers, argon ion lasers, or induced electric-arc discharges). The LPG spectral behavior consists of high attenuation rejection bands with a full width at half maximum (FWHM) of several nanometers. The spectral characteristics depend on the temperature, strain, curvature, and RI of the surrounding medium [3].

This multi-parameter sensing capability must be considered when they are to be used in the design of sensing devices. For the specific application of this work, temperature and RI variations are undesirable. However, if the medium surrounding the LPG does not change, the induced RI wavelength shifting does not present a relevant issue when working in an environment like a laboratory without significant temperature and humidity changes. On the other hand, temperature effects must be considered since the LPG temperature coefficients are around $0.154 \mathrm{~nm} /{ }^{\circ} \mathrm{C}$, and can have an impact on the final measurements [1]. Bhatia [1] showed that lower temperature sensitivities are achieved for longer grating periods, contrasting with higher sensitivities for curvature, RI and strain for shorter grating periods, so there should be suitable parameters for producing a LPG tuned for having higher sensitivities for curvature and strain sensing while diminishing the effects of temperature fluctuations [8].

At present, both strain and curvature sensors have been developed and vastly reported with sensitivities around $5.285 \mathrm{~nm} / \mathrm{m}^{-}$ ${ }^{1}$ and $49.3 \mathrm{pm} / \mathrm{m}^{-1}$ for curvature and up to $2 \mathrm{pm} / \mu \varepsilon$ and $1.2 \mathrm{pm} / \mu \varepsilon$ for strain, for LPGs and FBGs, respectively [9]-[13]. In this paper, it is reported and studied a simple mechanism to take advantage of the LPG curvature and strain sensitivity to perform high sensitivity micro-force measurements. A body of calibrated mass induces the curvature and strain, and the response of a stretched LPG and a precurvature LPG written in SMF-28 optical fiber to measure microforce is studied, in order to assess if an increase of sensitivity is achieved for the pre-curvature LPG.

\section{Materials and methods}

The electric-arc LPGs fabrication setup consists of a broadband light source (BBLS) connected at one end of the Corning SMF-28 optical fiber, and the other end connected to an Optical Spectrum Analyzer (OSA) to monitor the evolution of the LPG modes while in production. A DC discharge unit from a BICC AFS3100 optical fiber splice machine produces the markings, while a linear stage controller controls the grating period.

The produced LPG tracked mode chosen was the $\mathrm{LP}_{16}$ with a period of $395 \mu \mathrm{m}$, which provided a $1550 \mathrm{~nm}$ peak, monitored during a temperature sweep from $35.0^{\circ} \mathrm{C}$ to $99.8^{\circ} \mathrm{C}$. Its sensitivity to temperature was characterized with a connection to a BBLS (Thorlabs fiber-coupled SLD Source $2.5 \mathrm{~mW}$ ) and an OSA (Yokogawa AQ6370), while the grating was inserted inside an incubator (ESCO Isotherm incubation chamber) with a resolution of $\pm 0.1^{\circ} \mathrm{C}$, in a fixed position by two clamps, assuring no curvature induced changes. Thus, the temperature sensitivity of $109 \mathrm{pm} /{ }^{\circ} \mathrm{C}$ (in wavelength shifting) and $-0.07 \mathrm{~dB} /{ }^{\circ} \mathrm{C}$ (in amplitude variation) was measured.

Typical strain and curvature measurements were made using the setup presented in Fig. 1. This configuration allows the LPG to bend when the linear translation stage (LTS) is moved towards the fixed stage (FS), achieving curvature measurement. Strain measurement can be performed when the LTS is moved far away from the FS, stretching the optical fiber. A simple geometrical approach was used to calculate the curvature radius, R, only knowing its width $(w)$ and height (h), as shown in Fig. 1, by using the following equation:

$$
R=\frac{h}{2}+\frac{w^{2}}{8 h}
$$

Thus, the curvature, $C$, can be calculated by $C=1 / R$. To calculate the strain, the following equation can be used:

$$
\Delta \varepsilon=\frac{\Delta L}{L}
$$

where $\Delta L$ is the change in length, and $L$ is the original length.

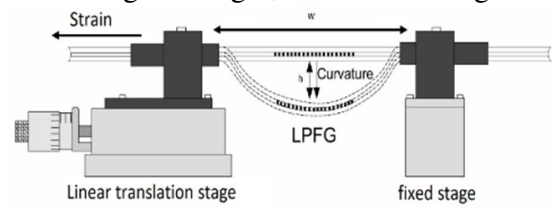

Fig. 1. Curvature and strain measurement setup. 
The initial spacing between the LTS and FS was $150 \mathrm{~mm}$, with the optical fiber stretched. For measuring curvature, displacement steps of $50 \mu \mathrm{m}$ were applied (moving the LTS towards the FS), while for measuring strain, displacement steps of $5 \mu \mathrm{m}$ were applied (moving the LTS away from the FS).

\section{Results and discussion}

Employing the LPG proposed in this work, curvature and strain sensitivities were measured to be $12.64 \mathrm{~nm} / \mathrm{m}^{-1}$ [Fig. 2(a)] and 0.47 $\mathrm{pm} / \mu \varepsilon$ [Fig. 2(b)], respectively. Force measurements were then performed by placing different masses on the center of the LPG, which was initially stretched (without pre-curvature). Although the contact with the mass induced a change in RI response towards lower wavelengths, these changes only resulted in a constant spectral offset, therefore not affecting the experiment. The weights of the masses produced forces (considering the earth gravitational acceleration constant of $9.806 \mathrm{~m} / \mathrm{s}^{2}$ ), which, in turn, induced both curvature and strain on the fiber, making these contributions to produce an effective wavelength shift increase (and both in the same direction). A set of masses ranging from $10 \mathrm{mg}$ to $500 \mathrm{mg}$ were hooked to the LPG, yielding the results presented in Fig. 2(c), with a sensitivity of $1.41 \mathrm{~nm} / \mathrm{mN}$.

In the curvature measurements [Fig. 2(a)], it is possible to see a linear behavior, and the middle of the studied range is around $2.2 \mathrm{~m}^{-}$ 1 . This value was thus chosen as an operation point on the microforce measurement with pre-curvature. Therefore, to test the LPG response, it was previously created a pre-curvature in it of $2.2 \mathrm{~m}^{-1}$, and then forces were applied by using different calibrated masses from $10 \mathrm{mg}$ to $300 \mathrm{mg}$, as presented in the experimental setup of Fig. 3. The results obtained are presented in Fig. 4(a), which was best fitted as a quadratic response (green curve). Regarding the linear range between $98 \mu \mathrm{N}$ and $686 \mu \mathrm{N}$ (red curve), the sensitivity obtained was $5.14 \mathrm{~nm} / \mathrm{mN}$, showing an enhancement of the precurvature LPG in comparison with the stretched LPG.

The spectral resolution, $r$, of this setup, can be estimated considering the values obtained from two measurements linked with two different forces, with the following expression [14]:

$$
r=\frac{2 \sigma}{S_{m}}
$$

where $\sigma$ is the highest standard deviation between the two steps, and $S_{m}$ is the sensitivity to mass variations. Regarding the stretched LPG, the resolution measurement is shown in Fig. 4(b).

The step of Fig. 4(b), between approximately $1524.30 \mathrm{~nm}$ and $1524.44 \mathrm{~nm}$, was obtained using two different forces $(9.8 \mu \mathrm{N}$ and $49 \mu \mathrm{N})$ and the results were acquired using the OSA set to its limiting resolution of $20 \mathrm{pm}$. The worst obtained standard deviation was $11 \mathrm{pm}$, the sensitivity $\left(S_{m}\right)$ was $1.41 \mathrm{~nm} / \mathrm{mN}$, resulting in a spectral resolution of $15.6 \mu \mathrm{N}$, for the worst-case (stretched LPG).

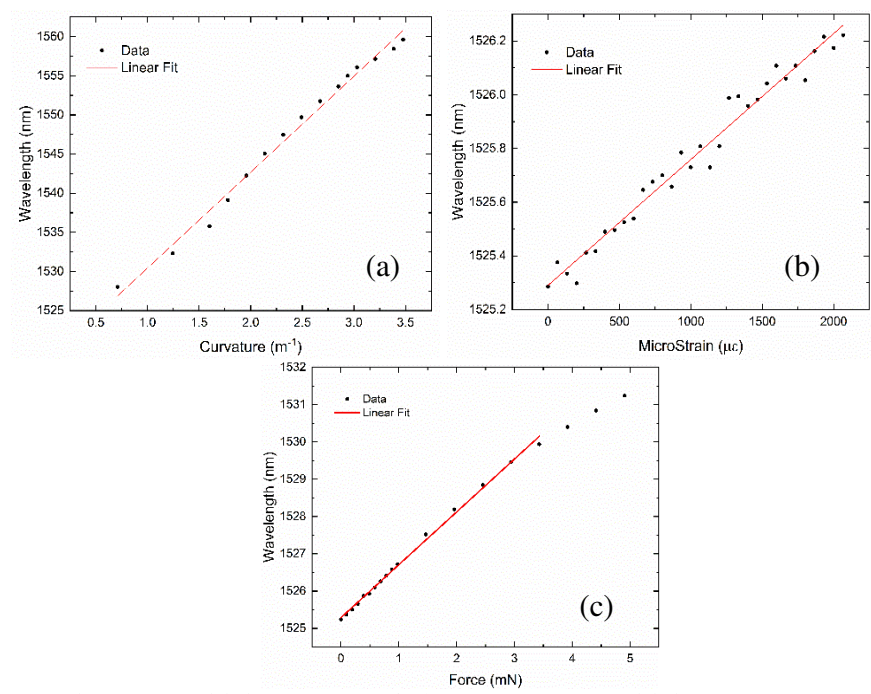

Fig. 2. Sensitivity curves of the LPG. (a) Sensitivity to curvature $(0.47 \mathrm{pm} / \mu \varepsilon)$. (b) Sensitivity to strain $\left(12.64 \mathrm{~nm} / \mathrm{m}^{-1}\right)$. (c) Sensitivity to force $(1.41 \mathrm{~nm} / \mathrm{mN})$.

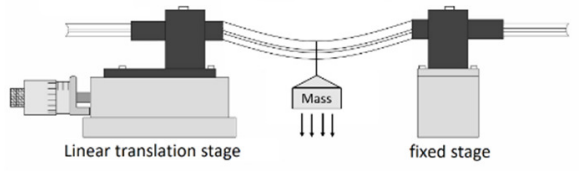

Fig. 3. Experimental setup to micro-force measurement with a precurvature LPG.
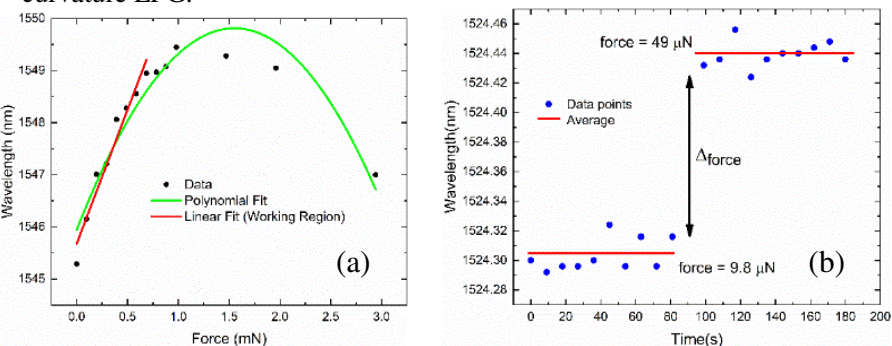

Fig. 4. Results of force measurement. (a) Sensitivity to force $\left(5.14 \mathrm{~nm} / \mathrm{mN}\right.$ ) for pre-curvature LPG (pre-curvature of $2.2 \mathrm{~m}^{-1}$ ). (b) Resolution measurement for forces of $9.8 \mu \mathrm{N}$ and $49 \mu \mathrm{N}$.

\section{Conclusions}

The LPG sensitivities to curvature, strain, and weight were measured to be $0.47 \mathrm{pm} / \mu \varepsilon, 12.64 \mathrm{~nm} / \mathrm{m}^{-1}$, and $1.41 \mathrm{~nm} / \mathrm{mN}$, respectively. Regarding the micro-force measurement, we obtained an enhancement from a sensitivity of $1.41 \mathrm{~nm} / \mathrm{mN}$ (stretched LPG) to $5.14 \mathrm{~nm} / \mathrm{mN}$ (pre-curvature LPG), a factor higher than 3 . Thereofore, with the proposed technique, based on using a precurvature LPG on the center of its linear range, one should be able to enhance the sensitivity of an LPG as a micro-force sensor.

\section{Acknowledgments}

The result presented in this paper is supported by grant \#2017/20128-2 and grant \#2019/04986-4, São Paulo Research Foundation (FAPESP) and financed in part by Coordenação de Aperfeiçoamento de Pessoal de Nível Superior - Brasil (CAPES) Finance Code 001, and is part of the Project "On Chip Whispering Gallery Mode Optical Microcavities For Emerging Microcontaminant Determination In Waters" - SAFE WATER, supported and co-funded by the European Commission, Directorate-General Communications Networks, Content and Technology (DG CONNECT) under the ERANET Cofund scheme - Horizon 2020 "Horizon 2020 - the Framework Programme for Research and Innovation (2014-2020)

\section{References}

[1] V. Bhatia, "Applications of long-period gratings to single and multi-parameter sensing," Opt. Express, vol. 4, no. 11, p. 457, 1999.

[2] X.-W. Zhao and Q. Wang, "Mini review: Recent advances in long period fiber grating biological and chemical sensors," Instrum. Sci. Technol., pp. 1-30, Sep. 2018.

[3] V. Bhatia and M. Vengsarkar, "Optical fiber long-period grating sensors.," Opt. Lett., vol. 21, no. 9, pp. 692-4, 1996.

[4] R. Pirich and K. D'Ambrosio, "Fiber optics for harsh environments," in 2011 IEEE Long Island Systems, Applications and Technology Conference, 2011, pp. 1-4.

[5] A. M. Vengsarkar, P. J. Lemaire, J. B. Judkins, V. Bhatia, T. Erdogan, and J. E. Sipe, "Long-period fiber gratings as band-rejection filters," J. Light. Technol., vol. 14, no. 1, pp. 58-64, 1996.

[6] G. Rego, “Arc-Induced Long Period Fiber Gratings,” J. Sensors, vol. 2016, 2016.

[7] T. Allsop et al., "Comparison between femtosecond laser and fusion-arc inscribed long period gratings in photonic crystal fibre," vol. 7357, p. 73570J, 2009.

[8] S. Chaubey, P. Joshi, M. Kumar, R. Arya, A. K. Nath, and S. Kher, "Design and development of long-period grating sensors for temperature monitoring," Sadhana Acad. Proc. Eng. Sci., vol. 32, no. 5, pp. 513-519, 2007.

[9] Q. Wang and Y. Liu, "Review of optical fiber bending/curvature sensor," Measurement, vol. 130, pp. 161-176, Dec. 2018.

[10] J. Kong, X. Ouyang, A. Zhou, H. Yu, and L. Yuan, "Pure Directional Bending Measurement With a Fiber Bragg Grating at the Connection Joint of Eccentric-Core and Single-Mode Fibers,” J. Light. Technol., vol. 34, no. 14, pp. 3288-3292, Jul. 2016.

[11] P. Geng et al., "Two-dimensional bending vector sensing based on spatial cascaded orthogonal long period fiber," Opt. Express, vol. 20, no. 27, p. 28557, Dec. 2012.

[12] R. Guyard, D. Leduc, C. Lupi, and Y. Lecieux, "Global overview of the sensitivity of long period gratings to strain,” Opt. Laser Technol., vol. 79, pp. 62-73, May 2016.

[13] R. J. Black, D. Zare, L. Oblea, Y.-L. Park, B. Moslehi, and C. Neslen, "ON THE GAGE FACTOR FOR OPTICAL FIBER GRATING STRAIN GAGES.”

[14] F. Chiavaioli et al., "Towards a Uniform Metrological Assessment of GratingBased Optical Fiber Sensors: From Refractometers to Biosensors," Biosensors, vol. 7, no. 4, p. 23, Jun. 2017. 